\title{
Global phenomenological and microscopic optical model potentials for alpha
}

\author{
Hairui $\mathrm{Guo}^{1}$, Xinwu $\mathrm{Su}^{2}$, Haiying Liang ${ }^{3}$, Yongli $\mathrm{Xu}^{2}$, Yinlu Han ${ }^{3, a}$, and Qingbiao $\mathrm{Shen}^{3}$ \\ 1 Institute of Applied Physics and Computational Mathematics, Beijing 100094, China \\ 2 College of Physics and Electronic Science, Shanxi Datong University, Datong 037009, China \\ 3 China Institute of Atomic Energy, PO Box 275(41), Beijing 102413, China
}

\begin{abstract}
The global phenomenological and microscopic optical model potentials for alpha with the incident energy up to $400 \mathrm{MeV}$ are obtained. The global phenomenological optical model potential is extracted by simultaneously fitting the experimental data of total reaction cross sections and elastic scattering angular distributions in the mass range of target nuclei $20 \leq A \leq 209$. The microscopic optical model potential is obtained by the Green function method through nuclear matter approximation and local density approximation based on the effective Skyrme nucleon-nucleon interaction. Both optical model potentials are used to calculate the reaction cross sections and elastic scattering angular distributions for the target nuclei in the mass range $12 \leq A \leq 209$ at incident alpha energies up to $400 \mathrm{MeV}$. The calculated results are compared with the experimental data, and the calculated results by phenomenological and microscopic optical model potentials are also compared with each other.
\end{abstract}

\section{Introduction}

Optical model is one of the most important theoretical models in nuclear reaction theory, which is widely used in the nuclear reaction analysis. The central assumption of this model is that the complicated interaction between an incident particle and a target nucleus is represented by a complex mean-field potential named optical model potential (OMP). A good optical model potential can be used to reliably predict the observables in the energy and nucleus region where no measurement data exist. The global phenomenological optical model potential (GOP) is obtained by adjusting its parameters to minimize the deviation between the calculated results and the experimental data of reaction cross sections and elastic scattering angular distributions in a wide energy and nucleus range. The microscopic optical model potential (MOP) is generated theoretically based on the nucleonnucleon interaction. Both OMPs have some prediction ability for the reactions with no measurement data in a wide energy and nucleus region.

The nuclear reactions with $\alpha$ incidence and $\alpha$ emission are of importance in nuclear astrophysics, nuclear engineering, and nuclear medicine. The $\alpha$ OMP is an essential and powerful tool for the understanding of such nuclear reactions, such as for the analysis of the $\alpha$ elastic scattering, the calculation of $\alpha$-induced reaction cross sections as well as the $\alpha$ production from compound reactions. Since the experimental data of such reactions are not sufficient, a global phenomenological OMP and a microscopic OMP for the incident $\alpha$ energy up to $400 \mathrm{MeV}$ and target nuclei in the mass range $12 \leq A \leq 209$ are provided here and used to predict the observables.
In Sect. 2, the formulations of both optical model potentials are given briefly. Section 3 is the analysis and comparison of the calculated results and experimental data. Finally, Sect. 4 is a summary.

\section{Formulation}

\subsection{Global phenomenological optical model potential}

The global phenomenological optical model potential is obtained by fitting the experimental data of total reaction cross sections and elastic scattering angular distributions for target nuclei with mass number $20 \leq A \leq 209$ below $400 \mathrm{MeV}$. The optical model potential is Woods-Saxon [1] form for the real part and the imaginary part corresponding to volume absorption, derivative Woods-Saxon form for the imaginary part corresponding to the surface absorption. The spin-orbit part is 0 , because the spin of the incident $\alpha$ is 0 . The optical model potential is expressed as

$$
V(r)=V_{r}(r)+i\left[W_{s}(r)+W_{v}(r)\right]+V_{c}(r),
$$

where $V_{r}(r)$ is the real part potential, $W_{s}(r)$ and $W_{v}(r)$ are the imaginary part potential corresponding to surface absorption and volume absorption, respectively, and $V_{c}(r)$ is the coulomb potential.

The energy dependencies of potential depths are expressed as follows:

The real part of optical potential:

$$
V_{r}=V_{0}+V_{1} E+V_{2} E^{2}+V_{3}(N-Z) / A+V_{4} Z A^{\frac{1}{3}}
$$

The imaginary part corresponding to the surface absorption:

$$
W_{s}=\max \left\{0, W_{0}+W_{1} E+W_{2}(N-Z) / A\right\}
$$

a e-mail: hanyl@ciae.ac.cn 
Table 1. Global optical model potential parameters for alpha.

\begin{tabular}{cc|cc}
\hline \hline $\mathrm{V}_{0}$ & 175.0881 & $\mathrm{a}_{r}$ & 0.6578 \\
$\mathrm{~V}_{1}$ & -0.6236 & $\mathrm{a}_{s}$ & 0.6359 \\
$\mathrm{~V}_{2}$ & 0.0006 & $\mathrm{a}_{v}$ & 0.5587 \\
$\mathrm{~V}_{3}$ & 30.000 & $\mathrm{a}_{s o}$ & 0.85 \\
$\mathrm{~V}_{4}$ & -0.2360 & $\mathrm{r}_{r}$ & 1.3421 \\
$\mathrm{~W}_{0}$ & 27.5816 & $\mathrm{r}_{s}$ & 1.2928 \\
$\mathrm{~W}_{1}$ & -0.0797 & $\mathrm{r}_{v}$ & 1.4259 \\
$\mathrm{~W}_{2}$ & 48.00 & $\mathrm{r}_{s o}$ & 1.2686 \\
$\mathrm{U}_{0}$ & -4.0174 & $\mathrm{r}_{C}$ & 1.35 \\
$\mathrm{U}_{1}$ & 0.1409 & & \\
\hline \hline
\end{tabular}

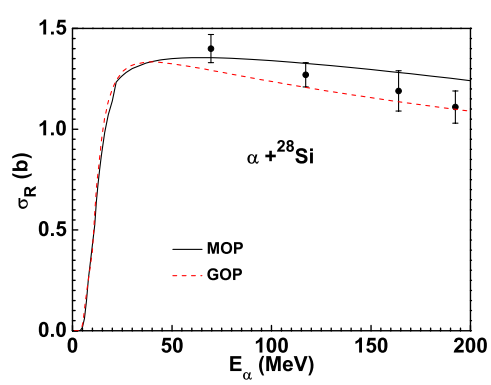

Figure 1. Comparison between the calculated reaction cross sections and experimental data (symbols) for $\alpha+{ }^{28} \mathrm{Si}$ reaction. The result calculated by MOP is denoted by the solid line, while that calculated by GOP is denoted by the dashed line.

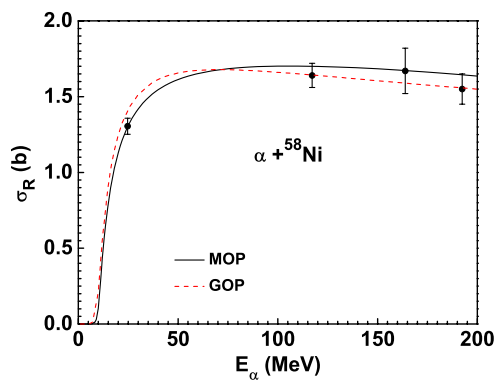

Figure 2. Same as Fig. 1 but for ${ }^{58} \mathrm{Ni}$.

The imaginary part corresponding to the volume absorption:

$$
W_{v}=\max \left\{0, U_{0}+U_{1} E\right\}
$$

where $Z, N$ and $A$ stand for charge, neutron and mass numbers of target, respectively; $E$ is the incident neutron energy in the laboratory system. The radius parameters of the real part, the surface absorption and the volume absorption are $r_{r}, r_{s}$ and $r_{v}$, respectively. The diffuseness widths of the real part, the surface absorption and the volume absorption are $a_{r}, a_{s}$ and $a_{v}$, respectively. The unit of the potential depth and the energy $E$ is $\mathrm{MeV}$, and the unit of the radius and diffuseness width is $f m$.

The optical model potential parameters are given in Ref. [2] as in Table 1.

\subsection{Microscopic optical model potential}

The microscopic optical model potential for $\alpha$ particle is generated by the Green function method based on the Skyrme nucleon-nucleon interaction. The detail of the formulation is described in Ref. [3]. From the view of the

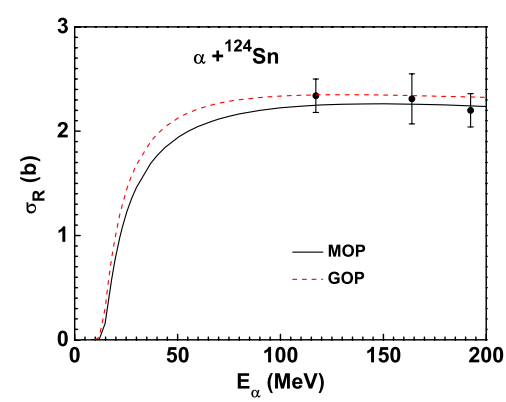

Figure 3. Same as Fig. 1 but for ${ }^{124} \mathrm{Sn}$.

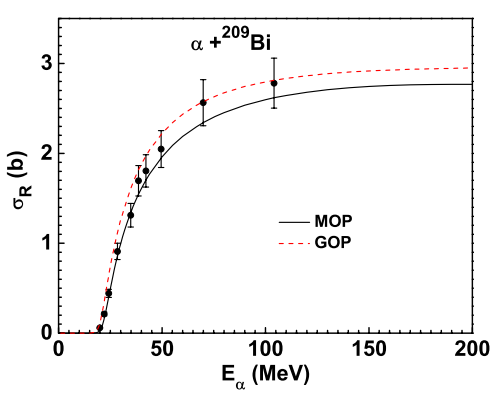

Figure 4. Same as Fig. 1 but for ${ }^{208} \mathrm{~Pb}$.
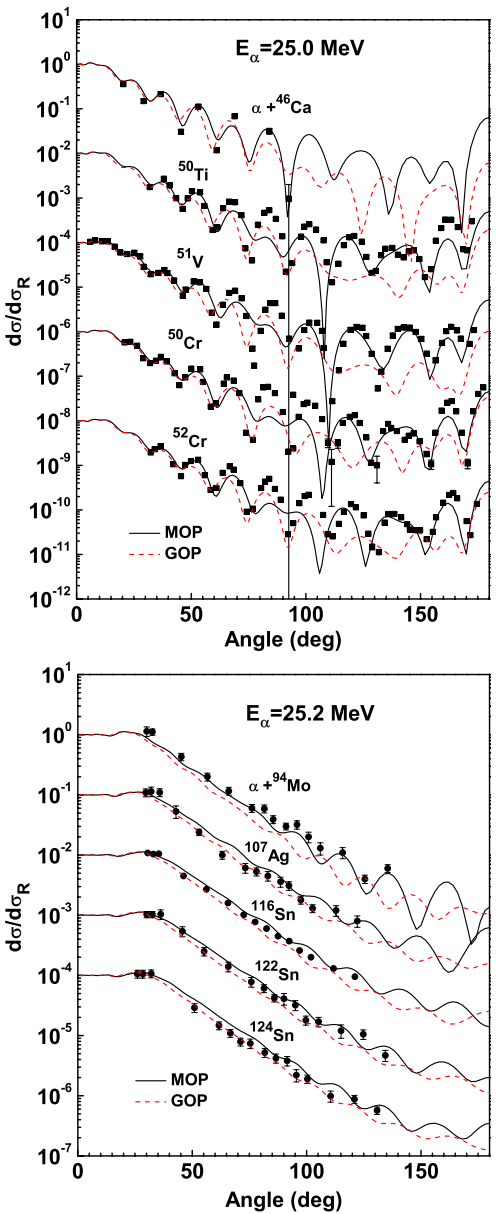

Figure 5. Comparison of the calculated elastic scattering angular distributions with experimental data (symbols) for different target nuclei at $25 \mathrm{MeV}$. The result calculated by MOP is denoted by the solid lines, while that calculated by GOP is denoted by the dashed lines. 

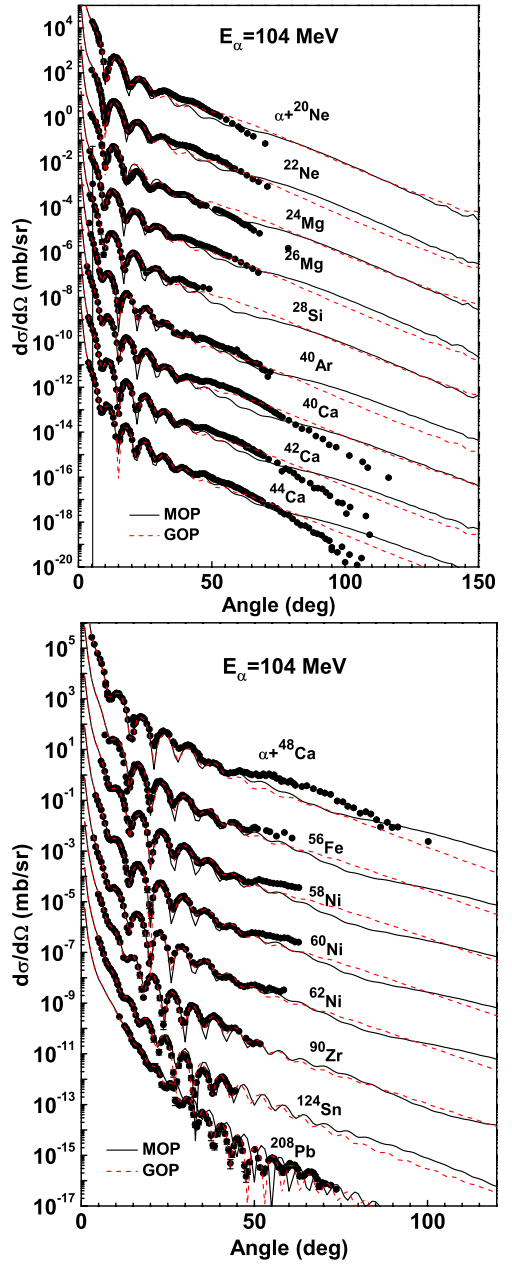

Figure 6. Same as Fig. 5 but at $104 \mathrm{MeV}$.

many-body theory, the optical potential can be identified with the mass operator of Green function. The first and second order mass operators of the four-particle Green function are derived in nuclear matter first. The firstorder mass operator is used to denote the real part of the microscopic optical model potential, and the imaginary part of second-order mass operator is used to denote the imaginary part potential. The microscopic optical model potential for a finite target nucleus is then obtained by applying a local density approximation. The densities of the neutron and proton in the target nucleus are expressed by Negele's formula [4]. With a version of the Skyrme interaction, GS2 [5], the microscopic optical potential for $\alpha$ particle is obtained numerically.

\section{Results and discussion}

The $\alpha$ MOP and GOP obtained are used to predict the reaction cross sections and elastic scattering angular distributions in the target nucleus range from ${ }^{12} \mathrm{C}$ to ${ }^{209} \mathrm{Bi}$ for incident energies up to $400 \mathrm{MeV}$. In most cases, The calculation can reproduce the experimental data reasonably well. The following gives some examples.

The calculated $\alpha$ reaction cross sections for ${ }^{28} \mathrm{Si}$, ${ }^{58} \mathrm{Ni},{ }^{124} \mathrm{Sn}$ and ${ }^{208} \mathrm{~Pb}$ are compared with the experimental data [6] in Figs. 1-4. The results calculated by MOP are denoted by the solid lines, while those calculated by GOP are denoted by the dashed lines. The results calculated by

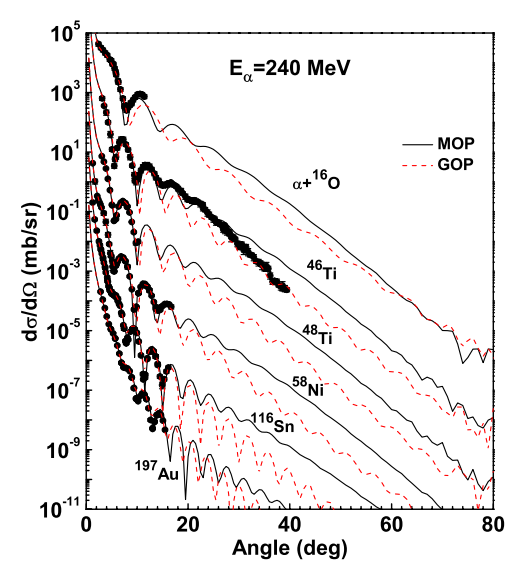

Figure 7. Same as Fig. 5 but at $240 \mathrm{MeV}$.

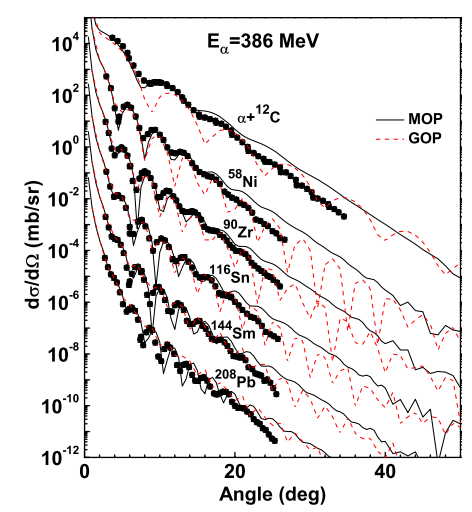

Figure 8. Same as Fig. 5 but at $386 \mathrm{MeV}$.

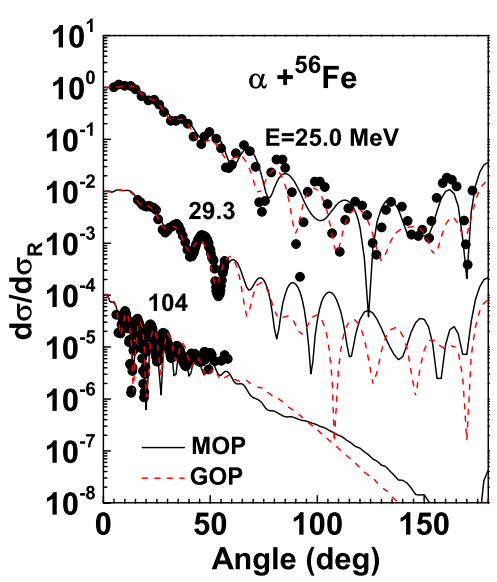

Figure 9. Comparison of the calculated $\alpha$ elastic scattering angular distributions with experimental data (symbols) for ${ }^{56} \mathrm{Fe}$. The result calculated by MOP is denoted by the solid lines, while that calculated by GOP is denoted by the dashed lines.

MOP is larger than those by GOP for relatively light nuclei, while smaller for relatively heavy nuclei. However, both calculated results are generally within the error bars of the experimental data.

The calculated results of elastic scattering angular distributions for different target nuclei are compared with the experimental data [7-14] at $25 \mathrm{MeV}, 104 \mathrm{MeV}$, $240 \mathrm{MeV}$ and $386 \mathrm{MeV}$ in Figs. 5-8, respectively. Both results calculated by MOP and GOP are in reasonable agreement with the experimental data in this wide energy 


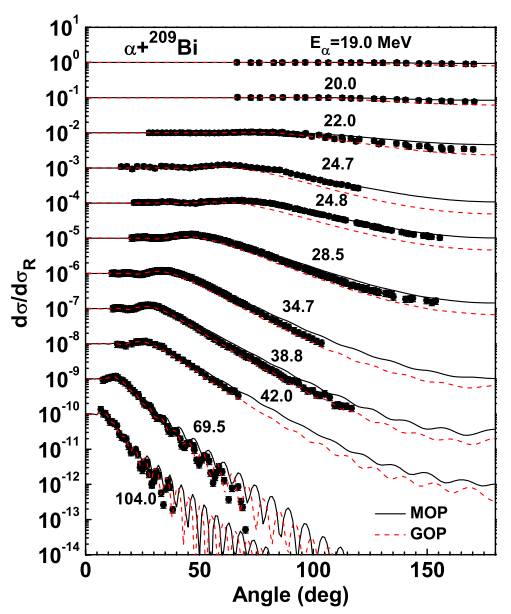

Figure 10. Same as Fig. 9 but for ${ }^{209} \mathrm{Bi}$.

region, and generally the GOP results are a little better than the MOP results in reproducing the experimental data.

Iron is one of the most important structure materials. The $\alpha$ elastic scattering angular distributions from ${ }^{56} \mathrm{Fe}$ calculated with the MOP and GOP are compared with the experimental data $[15,16]$ in Fig. 9. Both results reproduce the experimental data reasonably well.

The calculated result of the elastic scattering angular distribution for the heavy nucleus ${ }^{209} \mathrm{Bi}$ is also compared with the experimental data [17-19] in Fig. 10. Although the results calculated by MOP and GOP have some difference for back angles, both of them are in reasonable agreement with the experimental data.

\section{Summary}

An alpha global phenomenological optical potential is obtained by adjusting its parameters to fit the existing experimental data, and an alpha microscopic optical potential is obtained by the Green function method through nuclear matter approximation and local density approximation based on the GS2 Skyrme interaction. The calculated results of the reaction cross sections and elastic scattering angular distributions with both the GOP and MOP are generally in reasonable agreement with the experimental data for the target nuclei in the mass range $12 \leq A \leq 209$ at incident alpha energies up to $400 \mathrm{MeV}$. In some cases, the GOP is a little better than the MOP in reproducing the experimental data. Both of the optical model potentials may get direct application in some nuclear reaction analyses.

This work was supported by the National Natural Science Foundation of China under Grants numbers 11175260, 11347175, $11405099,11575291$.

\section{References}

[1] F.D. Becchetti and G.W. Greenlees, Phys. Rev. 182, 1190 (1969)

[2] X. Su and Y. Han, International Journal of Modern Physics E 24, 1550092 (2015)

[3] H. Guo, Y. Xu, H. Liang, Y. Han, and Q. Shen, Phys. Rev. C 83, 064618 (2011)

[4] J.W. Negele, Phys. Rev. C 1, 1260 (1970)

[5] S. Krewald, V. Klemt, J. Speth, and A. Faessler, Nucl. Phys. A 281, 166 (1977)

[6] A. Ingemarsson et al., Nucl. Phys. A 676, 3 (2000)

[7] F. Ballester et al., J. Phys. G 13, 1541 (1987)

[8] O.V. Bespalova et al., Izv. Ross. Akad. Nauk, Ser. Fiz. 56, 113 (1992)

[9] G. Hauser et al., Nucl. Phys. A 128, 81 (1969)

[10] H. Rebel et al., Nucl. Phys. A 182, 145 (1972)

[11] Y.W. Lui, H.L. Clark, and D.H. Youngblood, Phys. Rev. C 64, 064308 (2001)

[12] Y. Tokimoto, Y.W. Lui, H.L. Clark, B. John, X. Chen, and D.H. Youngblood, Phys. Rev. C 74, 044308 (2006)

[13] H.L. Clark, Y.W. Luib, and D.H. Youngblood, Nucl. Phys. A 589, 416 (1995)

[14] M. Uchida et al., Phys. Rev. C 69, 051301 (2004)

[15] F. Ballester et al., Nucl. Phys. A 501, 301 (1989)

[16] H.J. Gils et al., J. Phys. G 1, 344 (1975)

[17] A.R. Barnett and J.S. Lilley, Phys. Rev. C 9, 2010 (1974)

[18] P. Singh, A. Chatterjee, S.K. Gupta, and S.S. Kerekatte, Phys. Rev. C 43, 1867 (1991)

[19] A. Budzanowski et al., Phys. Lett. 11, 74 (1964) 